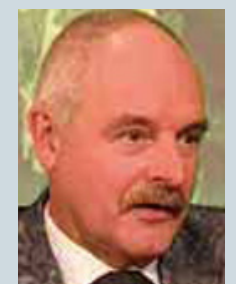

Prof. Dr. Klaus A. Milachowski Orthopädische Privatpraxis, Belegarzt an der Klinik Dr. Schreiber, München

\section{Individuelle Hüft-TEP: kein Vorteil in puncto Stress-Shielding}

Im Hinblick auf den periprothetischen Knochendichteverlust ist die "Unique“Hüftprothese, eine nach Maß angefertigte zementfreie Individualprothese, dem ebenfalls zementfreien Primärsystem ABG-I mit anatomisch adaptiertem Femurschaft offenbar nicht überlegen. In einer norwegischen Studie fand sich für beide Implantate ein teilweise erheblicher Substanzverlust, vor allem im proximalen Femur.

Individualprothesen sollen dank ihrer maßgeschneiderten Passform eine höhere Stabilität sowie eine verbesserte Krafteinleitung auf den proximalen Teil des Femurs erzielen. Das norwegische Team um Mona Nysted vom Trondheim University Hospital hat die mittelfristigen Knochendichteverluste nach Implantation des maßgeschneiderten "Unique“Systems mithilfe der DXA (Dual-energy X-ray absorptiometry) untersucht und die Ergebnisse mit einer Gruppe von Patienten verglichen, denen ein Standardsystem mit anatomisch adaptiertem $\mathrm{Fe}$ murschaft (ABG-I) implantiert worden war. Von den insgesamt 78 Patienten hatten 43 den Unique-Schaft, 35 das ABG-ISystem erhalten. Nachbeobachtet wurden die Studienteilnehmer fünf Jahre.

Die periprothetische Knochendichte (Bone Mineral Density, BMD) hatte global in beiden Gruppen über den gesamten Zeitraum deutlich abgenommen, in der Unique-Gruppe noch etwas stärker als in der ABG-Gruppe (11\% versus 7,7\%); allerdings war der Unterschied nicht signifikant $(p=0,09)$.

Am stärksten war die Reduktion in den Bereichen um den proximalen Prothesenschaft, den Gruen-Zonen 1 und 7. In Zone 1 betrugen die Verluste $15 \%$ in der ABG-I-Gruppe und $14 \%$ in der Unique-Gruppe. In der benachbarten Zone 7 hatte die BMD in beiden Grup- pen um $28 \%$ abgenommen. Geringer ausgeprägt, aber noch statistisch signifikant waren die Verluste von der Baseline in der ABG-Gruppe respektive in den Zonen 2, 3 und 4 in der Unique-Gruppe ( $\mathrm{p}<0,001)$.

Postoperativ hatte sich die BMD der Unique-Patienten zunächst deutlicher verringert als in der ABG-Gruppe. Als Grund nannten die Autoren, dass die Implantation des Unique-Schafts eine ausgedehntere Abtragung von Knochenmaterial erfordert. Diese Unterschiede schienen sich mit der Zeit durch RemodellingProzesse teilweise wieder auszugleichen, allerdings nicht im gewünschten Maße. Nach fünf Jahren war in der am distalsten gelegenen Zone 4 unterhalb der Implantatspitze ein statistisch signifikanter Unterschied zwischen den Gruppen auszumachen. Hier betrug der BMD-Verlust in der ABG-Gruppe 1,6\%, in der UniqueGruppe dagegen $9,7 \%(p=0,003)$. Sowohl präoperativ wie auch nach fünf Jahren waren die klinischen Scores in beiden Gruppen vergleichbar.

Fazit: Die zementfreie Unique-Individualprothese schneidet im Hinblick auf den Knochenabbau mittelfristig nicht besser ab als ein zementfreier anatomischer Standard-Schaft. In der periprothetischen Gruen-Zone 4 war der Knobis zum Jahr fünf in den Zonen 2 und 6

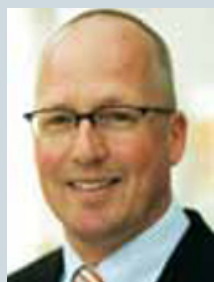

Dr. med. Stefan Middeldorf

Chefarzt

Orthopädische

Klinik,

Schön Klinik,

Bad Staffelstein chenerhalt beim ABG-Implantat sogar deutlich besser als beim Individualsystem. Nennenswerte klinische Auswirkungen hatte der Knochenverlust jedoch keine.

Dr. Elke Oberhofer

Nysted $\mathrm{M}$ et al. Periprosthetic bone loss after insertion of an uncemented, customized femoral stem and an anatomical stem. Acta Orthopaedica 2011;82(4):410-416

Kommentar: Dies ist eine interessante Studie, vor allem im Hinblick auf den aktuellen Trend zur "customized" Prothese, die durch immer besser informierte Patienten zunehmend gefordert und durch verschiedene Hersteller forciert wird. Besonders der signifikant höhere Knochenverlust distal der Implantatspitze ist bemerkenswert (1,6\% AGB versus $9,7 \%$ Unique). Hier stellt sich natürlich die Frage nach der Ursache, die in dieser Studie nicht abschließend beantwortet werden konnte. Durch die individualisierte Unique-Prothese ließ sich das Problem des proximalen stress-shieldings außerdem nicht nennenswert verbessern. Längerfristige Resultate müssen klären, ob höhere Fertigungskosten der Individualprothesen ihren Einsatz dauerhaft rechtfertigen können. Für den klinischen Alltag lieferte die Studie von Nysted und Kollegen interessante Ergebnisse, die einen Einfluss auf die Implantatwahl haben können.

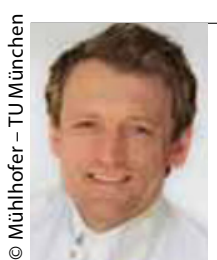

Dr. med. Heinrich Mühlhofer Klinik und Poliklinik für Orthopädie und Sportorthopädie

Klinikum rechts der Isar der TU München Ismaninger Straße 22 81675 München 